\title{
The role of parvalbumin and calbindin D28k in experimental scrapie
}

\author{
T. Voigtländer*, U. Unterberger*, M. Guentchev*1, B. Schwallert, M. R. Celio†, M. Meyer‡ and \\ H. Budka* \\ *Institute of Neurology, Medical University of Vienna, Vienna, Austria, †Unit of Anatomy, Department of Medicine, \\ University of Fribourg, Fribourg, Switzerland, and $¥$ Institute of Physiology, Department of Cellular Physiology, Ludwig \\ Maximilians University, Munich, Germany
}

\begin{abstract}
Aims: Prion diseases are generally characterized by pronounced neuronal loss. In particular, a subpopulation of inhibitory neurones, characterized by the expression of the calcium-binding protein parvalbumin (PV), is selectively destroyed early in the course of human and experimental prion diseases. By contrast, nerve cells expressing calbindin D28k (CB), another calcium-binding protein, as well as PV/CB coexpressing Purkinje cells, are well preserved. Methods: To evaluate, if PV and CB may directly contribute to neuronal vulnerability or resistance against nerve cell death, respectively, we inoculated PV- and CB-deficient mice, and corresponding controls, with 139A scrapie and compared them with regard to incubation times and histological lesion profiles. Results: While survival times were slightly but significantly diminished in CB-/-, but not PV-/- mice, scrapie lesion profiles did
\end{abstract}

not differ between knockout mice and controls. There was a highly significant and selective loss of isolectin $\mathrm{B}_{4}$-decorated perineuronal nets (which specifically demarcate the extracellular matrix surrounding the 'PVexpressing' subpopulation of cortical interneurones) in scrapie inoculated $\mathrm{PV}+/+$, as well as $\mathrm{PV}-/-$ mice. Purkinje cell numbers were not different in $\mathrm{CB}+/+$ and $\mathrm{CB}-/-$ mice. Conclusions: Our results suggest that PV expression is a surrogate marker for neurones highly vulnerable in prion diseases, but that the death of these neurones is unrelated to PV expression and thus based on a still unknown pathomechanism. Further studies including the inoculation of mice ectopically (over)expressing $\mathrm{CB}$ are necessary to determine whether the shortened survival of $\mathrm{CB}-/-$ mice is indeed due to a neuroprotective effect of this molecule.

Keywords: calbindin, calcium binding protein, neuroprotection, parvalbumin, prion disease, selective neuronal vulnerability

\section{Introduction}

Prion diseases are fatal neurodegenerative disorders of infectious, inherited or sporadic origin. They occur

Correspondence: Dr Till Voigtländer, Institute of Neurology, Medical University of Vienna, AKH 4J, Währinger Gürtel 18-20, A-1097 Vienna, Austria. Tel: +43 140400 5517; Fax: +43 140400 5511; E-mail: till.voigtlaender@meduniwien.ac.at

${ }^{1}$ Present address: Department of Neurosurgery, University Hospital St. Ivan Rilski, Sofia, Bulgaria. in humans as Creutzfeldt-Jakob disease, GerstmannSträussler-Scheinker disease, fatal familial insomnia and kuru, as well as in animals, like scrapie in sheep and bovine spongiform encephalopathy in cattle [1]. Damage to the central nervous system in prion diseases is typically characterized by a triad comprising spongiform change, astrogliosis and neuronal loss. In addition, an abnormal, partially protease-resistant isoform [(pathological isoform of the prion protein $\left.\left(\mathrm{PrP}^{\mathrm{Sc}}\right)\right]$ of the cellular prion protein $\left(\operatorname{PrP}^{\mathrm{C}}\right)$ accumulates mainly in the brain of affected 
individuals. Neither the precise nature of the infectious agent nor the central nervous pathomechanisms have so far been fully elucidated [2-4].

In an attempt to characterize increased susceptibility or resistance of distinct neuronal subpopulations to prionrelated neurodegeneration, we previously determined the survival of calbindin D28k (CB) and parvalbumin (PV) containing neurones in different types of prion diseases. We found an early, selective and severe, but incomplete loss of PV-positive inhibitory interneurones in the frontal, temporal and entorhinal cortex, and in the preparasubiculum in humans, as well as in the retrosplenial granular cortex in mice, while $\mathrm{CB}$ expressing interneurones, like PV/CB coexpressing Purkinje cells, were well preserved [5-7].

Parvalbumin and $\mathrm{CB}$, which belong to the EF-hand family of calcium-binding proteins, are thought to mainly serve as cytosolic calcium buffers, but for $\mathrm{CB}$ additional roles as a calcium sensor have been reported [8]. In the brain, they are expressed in specific, mainly nonoverlapping subsets of neurones $[9,10]$. The exact mode of how these proteins modify temporal and spatial aspects of $\mathrm{Ca}^{2+}$ transients to maintain intracellular $\mathrm{Ca}^{2+}$ homeostasis remained obscure until knockout mice were generated $[9,11]$. Most surprisingly, null mutant strains ( $\mathrm{CB}-/-$ and PV-/-) show a normal development in terms of life span, growth, fertility, physical activity and behaviour, under standard housing conditions $[9,11,12]$; in specific test cages, however, subtle alterations in locomotor activity have recently been reported for both genotypes [13]. Furthermore, on the light microscopic level, no differences in brain morphology were observed at any age between mutant and wild-type mice $[9,12]$. Due to their $\mathrm{Ca}^{2+}$ buffering capacity, $\mathrm{CB}$ and $\mathrm{PV}$ have been discussed to influence survival or death of neurones under pathological conditions that are combined with a rapid increase of the intracellular $\mathrm{Ca}^{2+}$ load, either as neuroprotective molecules or as factors selectively increasing neuronal vulnerability. However, no generalized relationship between the expression of a specific calcium-binding protein on one hand, and its protective or antiprotective role on the other hand, has so far been established.

In prion diseases, PV may directly contribute to neuronal vulnerability, and $\mathrm{CB}$, vice versa, to the resistance against nerve cell death, by as yet unknown mechanisms. To evaluate a possible causal relationship, we inoculated PV- and CB-deficient mice, as well as corresponding controls, with the 139A scrapie strain and compared them with regard to incubation times and histological lesion profiles, the latter comprising spongiform change, astrogliosis and $\mathrm{PrP}^{\mathrm{sc}}$ deposition. In addition, the loss of cortical 'PV-interneurones' (that is interneurones that would normally contain PV) was quantified in PV knockout mice, and the general survival of Purkinje cells was analysed in CB-deficient animals, both in comparison with the respective control mice.

\section{Materials and methods}

\section{Animals and housing conditions}

The experimental set-up was approved by the national animal welfare committee at the federal ministry of health and women, Vienna. In the present experiment, 16 adult female $\mathrm{PV}-/-$ mice and $12 \mathrm{PV}+/+$ animals, as well as 26 adult female $\mathrm{CB}-/-$ mice and $13 \mathrm{CB}+/+$ animals were used. Generation and initial characterization of these null mutant strains of mice are described in detail elsewhere $[9,11]$. Homozygous breeding pairs for $\mathrm{PV}+/+$ and $\mathrm{PV}-/-$ mice both had a mixed 129/OlaHsd (129P2) × C57BL/6J (B6) genetic background [11], while the breeding pairs for $\mathrm{CB}+/+$ and $\mathrm{CB}-/-$ mice were based on a mixed 129R1 $\times$ B6 genetic background [9]. The animals were maintained under standard conditions (that is artificial day-night cycle with light from 6 a.m. to 6 p.m.; constant temperature/humidity). Separated according to their strain, mice were kept in groups of two to three animals in type II cages. In three independent sessions, $12 \mathrm{PV}-/$ - and six $\mathrm{PV}+/+$ mice (age 10-11 weeks), as well as $17 \mathrm{CB}-/-$ and seven $\mathrm{CB}+/+$ mice (age between 9 and 13 weeks) were inoculated intracerebrally (i.c.) with $30 \mu \mathrm{l}$ of the $139 \mathrm{~A}$ scrapie strain (provided by M. Groschup, Riems, Germany) at a 1:100 dilution. Concomitantly, four PV-/-, six PV+/+, nine $\mathrm{CB}-/-$ and six $\mathrm{CB}+/+$ control animals were inoculated (i.c.) with $30 \mu \mathrm{l}$ of noninfectious brain homogenate (mock) at the same dilution. To allow standardized sampling of brain tissue and, at the same time, determination of survival times in scrapie inoculated mice, spontaneous death was awaited in those animals, which were the first to develop the disease in each experimental group (that is one $\mathrm{PV}+/+$, four $\mathrm{PV}-/-$, three $\mathrm{CB}+/+$ and six $\mathrm{CB}-/-$ mice), while all other animals were killed with $\mathrm{CO}_{2}$ at the ultimate disease stage, where spontaneous death was expected to occur within less than a day. Of the scrapie inoculated CB-deficient mice, one animal died of an intercurrent infection 116 days after inoculation prior to the 
development of clinical symptoms of scrapie and was therefore excluded from further analysis. Controls were killed several days after the last scrapie animal of the respective experimental group. After death, animals were immediately perfused transcardially with $20 \mathrm{ml}$ phosphate buffered saline (PBS), followed by $20 \mathrm{ml} 4 \%$ buffered formalin. Subsequently, brains were removed, post-fixed for $24 \mathrm{~h}$ in $4 \%$ formalin and rinsed in PBS for several days. Coronal slices of forebrain and cerebellum were embedded in paraffin. For haematoxylin-eosin staining and immunohistochemical analysis, 4- $\mu \mathrm{m}$ sections were prepared.

\section{Immunohistochemistry and staining of perineuronal nets $(\mathrm{PNN})$}

For immunohistochemical analyses, monoclonal antibodies against CB-D28k (clone CL-300; Sigma-Aldrich Co., St Louis, MO, USA; dilution 1:50), PV (clone PA-235; SigmaAldrich Co.; 1:5000) and PrP (clone 6H4; Prionics AG, Schlieren-Zürich, Switzerland; 1:500), as well as a polyclonal antibody against glial fibrillary acidic protein (GFAP, Dako, Glostrup, Denmark; 1:3000), were applied. Peroxidase-labelled isolectin $\mathrm{B}_{4}\left(\mathrm{ILB}_{4}\right)$ from vicia villosa $(10 \mu \mathrm{g} / \mathrm{ml}$ in Tris buffered saline; Sigma-Aldrich Co.; 1:100) was used to decorate perineuronal nets (PNN). Pretreatment of paraffin sections included antigen retrieval by steaming for $60 \mathrm{~min}$ in citric acid buffer, pH $6.0\left(\mathrm{CB}, \mathrm{ILB}_{4}\right)$, antigen retrieval followed by incubation in concentrated formic acid for $2 \mathrm{~min}(\mathrm{PV})$, and digestion with $0.3 \mathrm{mg} / \mathrm{ml}$ protease type XXIV (Sigma-Aldrich Co.) in PBS for $10 \mathrm{~min}$ at $37^{\circ} \mathrm{C}$ (GFAP). Staining of $\mathrm{PrP}^{\mathrm{Sc}}$ was performed with a three-step pretreatment protocol comprising hydrated autoclaving, incubation in concentrated formic acid and, subsequently, in guanidine isothiocyanate. Primary antibodies were diluted in a detergentcontaining diluent (Dako) and incubated overnight at $4^{\circ} \mathrm{C}$. The ChemMate ${ }^{\mathrm{TM}}$ Detection Kit (Dako) was used for visualization according to the manufacturer's protocol. The specificity of primary antibodies was verified by appropriate serum and isotype controls. For lectin staining, sections were incubated with peroxidase-labelled $\mathrm{ILB}_{4}$ for $1 \mathrm{~h}$ at $37^{\circ} \mathrm{C}$ and developed with diaminobenzidine.

\section{Lesion profiling}

Classical mouse scrapie lesion profiles (degree of spongiform change in the neuropil and white matter tracts, as well as neuronal vacuolation) were determined according to the method described by Fraser and Dickinson [14] and Bruce et al. [15]. Briefly, vacuolar degeneration was scored on a scale of 0 (no vacuolar degeneration) to 5 (massive, confluent vacuolation with an average number of 300400 vacuoles per analysed microscopic field) in nine defined grey matter and three white matter areas (for details see legend of Figure 2) using a Nikon photomicroscope equipment (microscope: Nikon Eclipse E600, objective: Nikon Plan Apo 40 ×/0.95 DIC M; Nikon corporation, Tokyo, Japan). In order to assess predefined brain areas properly, an average score for 2-6 neighbouring high power fields (field size $200 \times 140 \mu \mathrm{m}$ ) was determined for each of the brain regions. Apart from the analysis of spongiform degeneration, we also investigated the extent and distribution of $\mathrm{PrP}^{\mathrm{Sc}}$ deposition and gliosis semiquantitatively in the same grey matter areas that were used for the classical lesion profiling. For this purpose, we used the following scores: (i) $\operatorname{PrP}^{\mathrm{sc}}$ : 0 (no deposition), 1 (mild deposition), 2 (moderate deposition) and 3 (massive deposition); (ii) GFAP: 0 (no astrogliosis), 1 (single reactive fibrillary astrocytes within the neuropil), 2 (moderate number of reactive fibrillary astrocytes), 3 (moderate to high number of reactive fibrillary astrocytes and strong background of GFAP positive astrocytic processes in the neuropil), 4 (high number of reactive astrocytes with transition features towards gemistocytic astrocytes) and 5 (high number of highly reactive gemistocytic astrocytes) (representative pictures for GFAP and $\mathrm{PrP}^{\mathrm{Sc}}$ are provided as electronic supplementary material in Figures S1 and S2 respectively).

The number of normally PV-expressing cortical interneurones was quantified by light microscopy (Nikon Plan Apo $60 \times / 0.95$ DIC M) in scrapie, as well as mock, inoculated $\mathrm{PV}-/-$ and $\mathrm{PV}+/+$ mice by counting nerve cells surrounded by $\mathrm{ILB}_{4}$-decorated $\mathrm{PNN}$ in the agranular and granular retrosplenial cortex of both hemispheres at the level of the thalamus (brain areas according to Paxinos and Franklin [16]). Only neurones with clearly visible cytoplasm and nucleus were included in the statistical evaluation.

Finally, the density of Purkinje cells was analysed in scrapie infected $\mathrm{CB}-/-$ and $\mathrm{CB}+/+$ mice by determining the total number of Purkinje cells, as well as the average number of Purkinje cells per high power field, in 20 high power fields (field length $147 \times 103 \mu \mathrm{m}$ ) in the second and third cerebellar lobule (later referred to as region A) and 12 high power fields in the lateral portion of the third and fourth cerebellar lobules, the simple lobule, and crus 1 
of the ansiform lobule (later referred to as region B) using a $60 \times$ objective (brain areas see [16]). All assessments were performed in a diagnosis-blinded fashion.

\section{Statistics}

Significances of differences in survival times between different groups of animals were evaluated with the log rank test. The number of $\mathrm{ILB}_{4}$-decorated PNN in scrapie and mock inoculated PV+/+ and PV-/- mice, as well as the density of Purkinje cells in the cerebellar cortex in scrapie and mock inoculated $\mathrm{CB}+/+$ and $\mathrm{CB}-/-$ mice were compared by means of the Mann-Whitney test. Statistical analyses were carried out with the sPSs 10.0 (SPSS Inc. Chicago, IL, USA) and the StatGraphics 4.0 software (StatPoint, Inc., Herndon, VA, USA).

\section{Results}

\section{Survival times are diminished in scrapie inoculated $\mathrm{CB}-/-$ mice as compared with controls}

Deficiency for CB shortened survival after infection with scrapie prions to a small, but highly significant degree. Scrapie infected $\mathrm{CB}+/+$ mice died at $160.0 \pm 3.1$ days (mean \pm standard deviation), while scrapie inoculated CB-/- mice only survived for $150.4 \pm 6.7$ days after i.c. inoculation $(P=0.0002$; Figure 1B). Of note, all except one CB knockout mice had died or had to be sacrificed as a result of progression to the final disease stage before the first control died due to scrapie (Figure 1B). By contrast, the presence or absence of PV did not significantly influence survival times in scrapie animals. $\mathrm{PV}+/+$ mice died at $156.2 \pm 9.8$ days, $\mathrm{PV}-/-$ mice at $159.1 \pm 7.9$ days after inoculation $(P=0.62$; Figure $1 \mathrm{~A})$.

\section{Scrapie lesion profiles do not differ between $\mathrm{CB}-/-$, as well as $\mathrm{PV}-/-$ mice and their respective controls}

Evaluating the classical lesion profile, which describes the degree of spongiform change in neuropil and nerve cells $[14,15]$, we did not observe a significant difference between scrapie infected PV- or CB-deficient mice and their respective wild-type controls (Figures 2A,D and 3A,D,G,K). The lesion profile in all groups was consistent with the 'reference profile' reported by Bruce et al. [15]. Noteworthily, in the grey matter, lesion scores were in most instances higher in the rostral part of the brain (scoring positions 5-9; Figure 2A,D) as compared with the more caudal parts (scoring positions 1-4, Figure 2A,D). The highest scores were found in the thalamus, the paraterminal body and the anterior paramedian cerebral cortex (positions 5, 7, 9), while the lowest score was present in the cerebellar cortex (position 2).
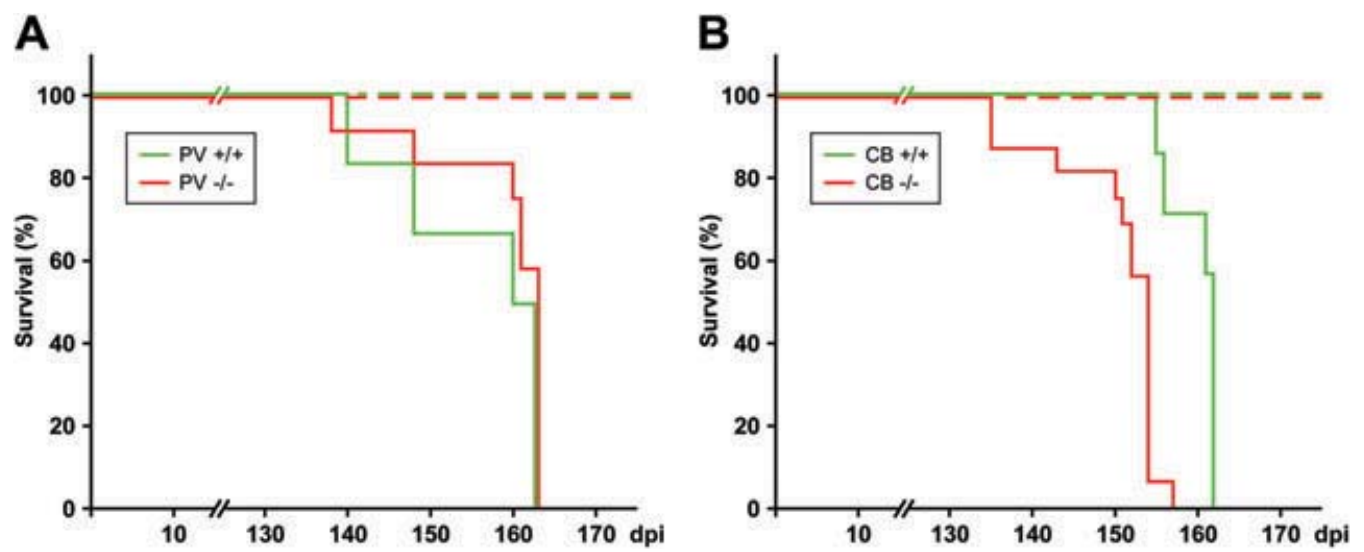

Figure 1. Kaplan-Meier survival analysis of parvalbumin (PV) and calbindin D28K (CB) deficient, as well as PV+/+ and CB+/+ wild-type mice intracerebrally inoculated with the 139A scrapie strain. (A) Survival times of scrapie infected PV-/- animals are shown by the red, those of scrapie inoculated $\mathrm{PV}+/+$ mice by the green solid line, demonstrating no significant difference between the two strains of mice. The red- and green-dashed lines represent mock inoculated PV-/- and PV+/+ control animals respectively. (B) By contrast, comparison of the survival times of scrapie inoculated CB-/- (red solid line) and CB+/+ mice (green solid line) reveals a small, albeit highly significant difference between the two strains of mice. All except one $\mathrm{CB}$ knockout mice had died or had to be sacrificed as a result of progression to the final disease stage before the first control died due to scrapie. Red- and green-dashed lines represent mock inoculated $\mathrm{CB}-/-$ and $\mathrm{CB}+/+$ control animals respectively. 

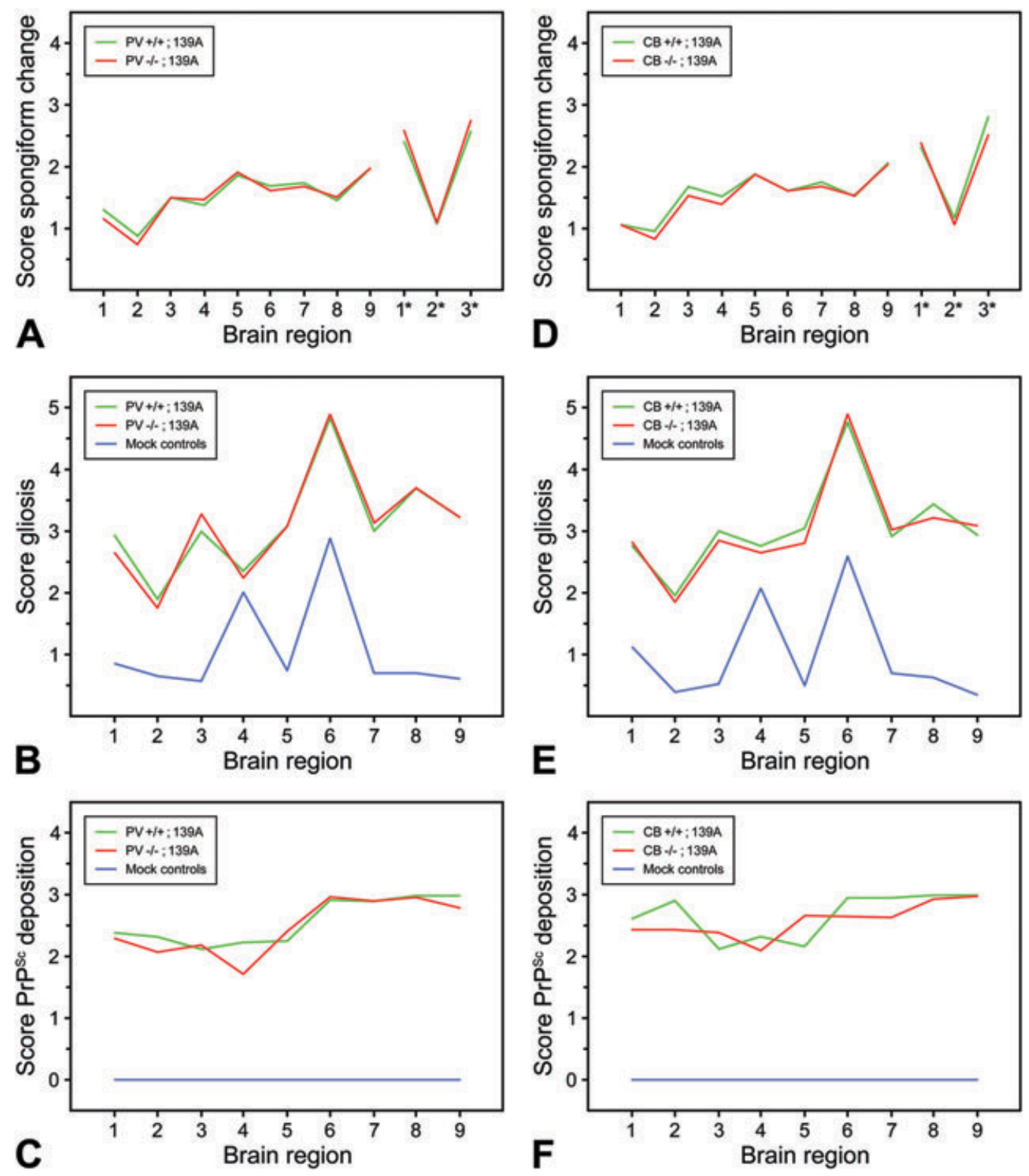

Figure 2. Lesion profiles illustrating vacuolar degeneration, astrogliosis and pathological isoform of the prion protein $\left(\operatorname{PrP}^{\mathrm{Sc}}\right)$ deposition of parvalbumin (PV) and calbindin D28K (CB) deficient mice, as well as corresponding $\mathrm{PV}+/+$ and $\mathrm{CB}+/+$ controls intracerebrally inoculated with 139A scrapie. Almost congruent lesion profiles are observed in grey matter (A-F) and white matter areas (A,D) when PV-/- (red line) and $\mathrm{PV}+/+$ mice (green line) (A-C) or CB-/- (red line) and CB+/+ mice (green line) (D-F) are compared with regard to the extent of vacuolar degeneration $(\mathbf{A}, \mathbf{D})$, reactive astrogliosis $(\mathbf{B}, \mathbf{E})$ and $\mathrm{PrP}^{\mathrm{Sc}}$ deposition $(\mathbf{C}, \mathbf{F})$. Blue lines in $(\mathbf{B})$ and $(\mathbf{E})$ illustrate the combined scores for reactive gliosis in mock-inoculated $\mathrm{PV}-/-$ and $\mathrm{PV}+/+(\mathrm{B})$, as well as $\mathrm{CB}-/-$ and $\mathrm{CB}+/+(\mathrm{E})$ healthy control mice. Note the relatively high control scores in the hypothalamus (4) and hippocampus (6), the latter presumably pioneering the peak scores in this area in the related scrapie infected animals. Analysed grey matter areas included: 1, dorsal medulla; 2, cerebellar cortex; 3, superior colliculus; 4 hypothalamus; 5 , medial thalamus; 6 hippocampus; 7 , paraterminal body; 8 , posterior medial cerebral cortex at the level of the thalamus; 9 , anterior medial cerebral cortex at the level of the paraterminal body (A-F). Analysed white matter areas included: $1^{*}$, cerebellar white matter; $2^{*}$, white matter of the mesencephalic tegmentum; $3^{*}$, pyramidal tract $(\mathrm{A}, \mathrm{D})[14,15]$. 

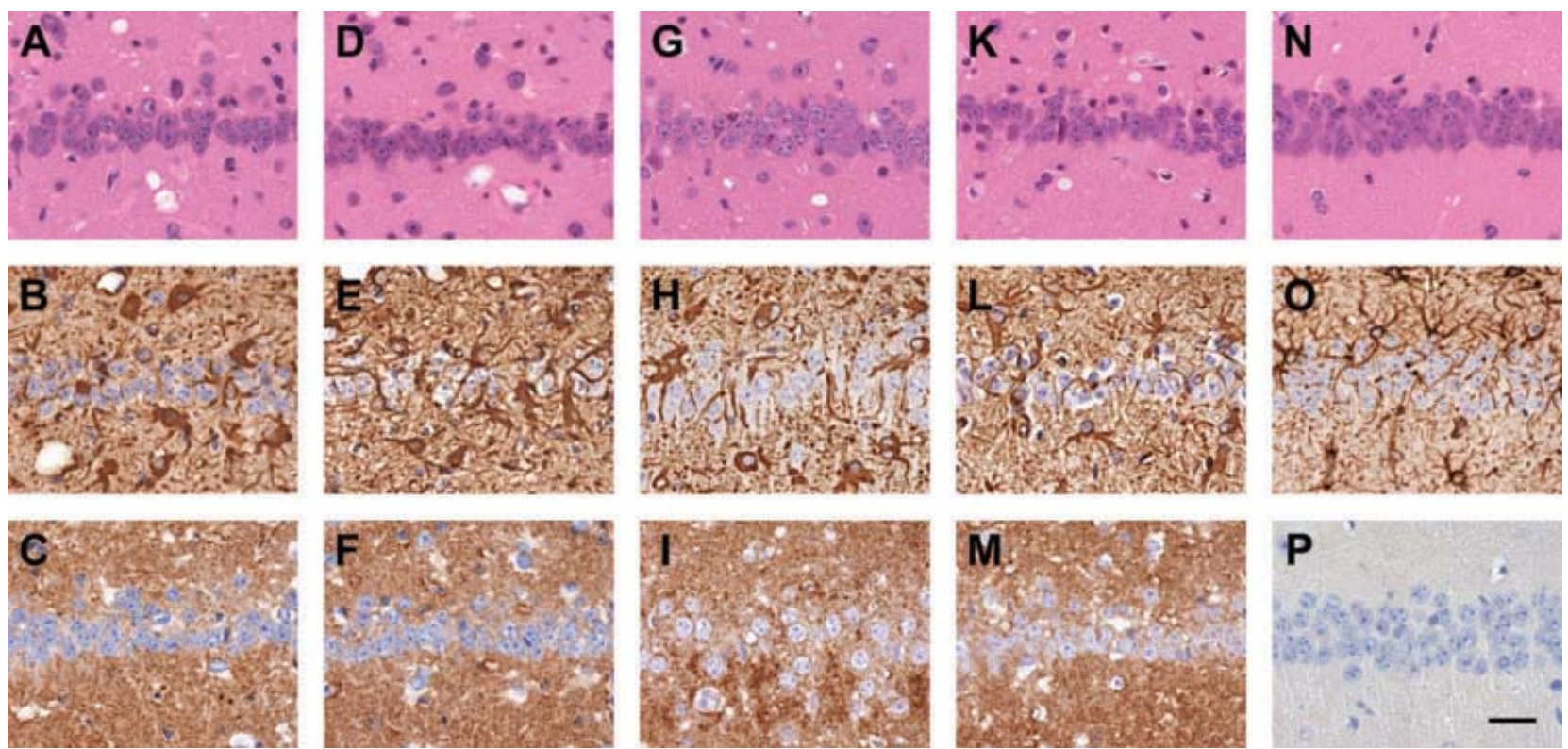

Figure 3. Comparison of vacuolar degeneration, astrogliosis and pathological isoform of the prion protein $\left(\mathrm{PrP}^{\mathrm{Sc}}\right)$ deposition as the key histopathological features of prion diseases between 139A scrapie inoculated parvalbumin (PV) and calbindin D28k (CB) deficient, as well as $\mathrm{PV}$ and $\mathrm{CB}$ expressing mice, and mock-inoculated control animals. (A- M) Photomicrographs displaying the extent of spongiform change (haematoxylin-eosin staining; A,D,G,K), astrogliosis [glial fibrillary acidic protein (GFAP) immunohistochemistry; B,E,H,L], and PrP ${ }^{\text {Sc }}$ deposition ( $\mathrm{PrP}^{\mathrm{Sc}}$ immunohistochemistry; C,F,I,M) in the CA1 region of the hippocampus of a representative scrapie infected PV-/- (A- C), $\mathrm{PV}+/+(\mathrm{D}-\mathrm{F}), \mathrm{CB}-/-(\mathrm{G}-\mathrm{I})$, and $\mathrm{CB}+/+(\mathrm{K}-\mathrm{M})$ mouse. The histopathological changes in all three categories are uniform throughout the four strains of mice. (N-P) Conventional (haematoxylin-eosin; N) and immunohistochemical (GFAP, PrP ${ }^{\mathrm{Sc}} ; \mathrm{O}, \mathrm{P}$ ) staining of the same region of one representative control animal inoculated intracerebrally with mock brain homogenate. Noteworthily, these animals consistently show a significant extent of reactive astrogliosis particularly in the hippocampus but no neuronal loss $(\mathbf{O})$. Scale bar in (P) corresponds to $25 \mu \mathrm{m}$.

When we determined the degree of astroglial activation in the same grey matter areas as defined for the classical lesion profile, we again did not detect any significant differences between all groups of scrapie inoculated mice (Figures 2B,E and 3B,E,H,L). As in the classical lesion profile, we observed a tendency for the degree of astrogliosis to be enhanced in the rostral areas of the brain (scoring positions 5-9) as compared with the caudal part (scoring positions 1, 2 and 4; Figure 2B,E). While the lowest score was again detected in the cerebellar cortex (position 2), the highest scores were found in the hippocampus and the posterior paramedian cerebral cortex at the level of the thalamus (positions 6 and 8). Importantly, mock inoculated animals show a low to medium degree of astroglial activation in the hippocampus and the hypothalamus (positions 6 and 4, Figure 2B,E: blue line; Figure 30); therefore, the peak score in the hippocampus in scrapie infected mice most likely represents an artefact due to the intracerebral inoculation route.

Finally, scoring of $\mathrm{PrP}^{\mathrm{sc}}$ deposition in the previously established grey matter areas [14] did not reveal any sig- nificant difference between scrapie infected animals of all genotypes (Figures 2C,F and 3C,F,I,M). Higher scores were again found in the rostral (positions 6-9), lower scores in caudal brain areas (positions 1-4, Figure 2C,F).

\section{Highly significant, selective loss of ILB4-decorated PNN in scrapie inoculated $\mathrm{PV}+/+$, as well as $\mathrm{PV}-/-$, mice}

In control mice, the average number of $\mathrm{ILB}_{4}$-decorated PNN (which specifically demarcate PV-positive cortical interneurones) in the retrosplenial cortex of one hemisphere dropped from 44.4 in mock injected healthy animals to 24.4 in scrapie inoculated mice, equivalent to a loss of $45.0 \%$ of PV-expressing inhibitory interneurones (Figure 4A,B,D). In PV-deficient mice, the average number of $\mathrm{ILB}_{4}$-decorated PNN decreased from 42.0 in mock inoculated controls to 25.8 in scrapie infected mice, corresponding to a loss of $38.6 \%$ of 'PV-interneurones' (Figure 4A,C). Statistical analysis revealed a highly significant difference in the average number of PNN between 

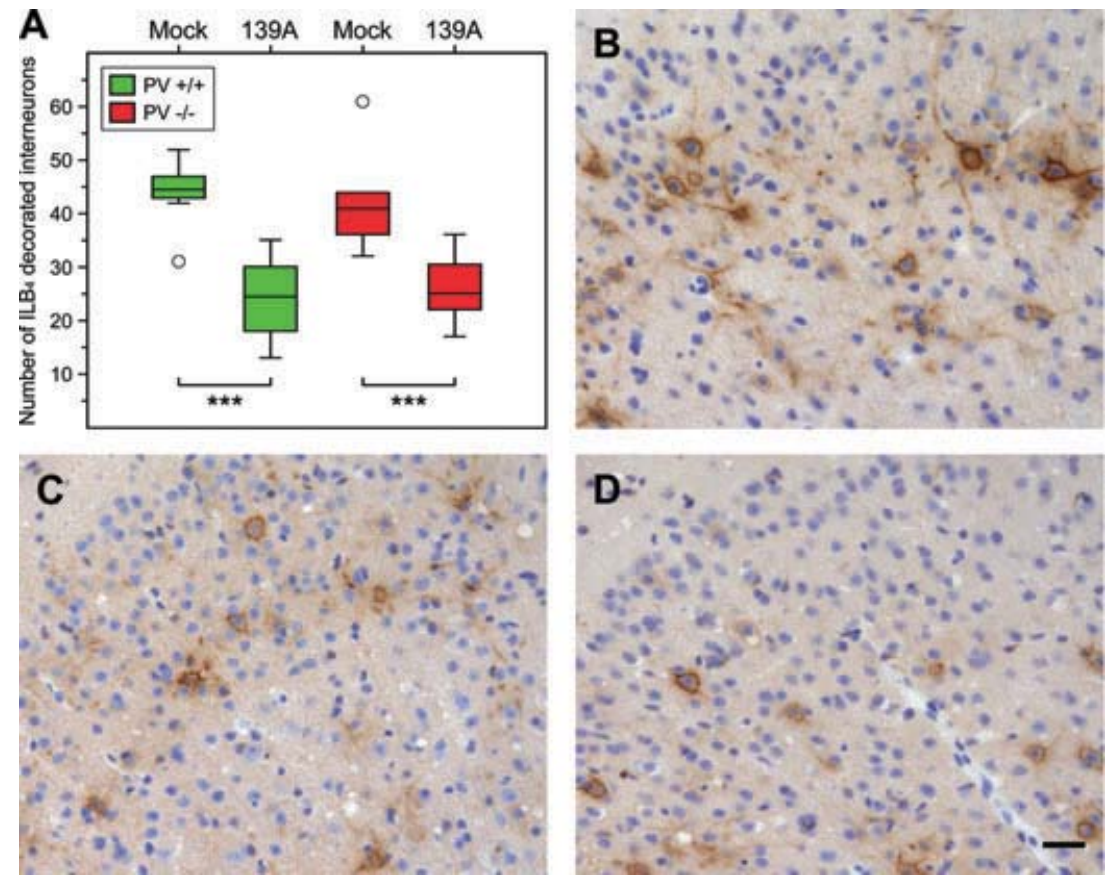

Figure 4. Selective vulnerability of physiologically parvalbumin (PV) expressing, isolectin $\mathrm{B}_{4}\left(\mathrm{ILB}_{4}\right)$ surrounded cortical interneurones in 139A scrapie inoculated PV deficient mice and PV expressing controls. (A) Highly significant loss of ILB $_{4}$ decorated interneurones in the granular and agranular retrosplenial cortex in 139A scrapie inoculated PV-/- (red boxes) and PV+/+ mice (green boxes) as compared with the related control animals [cell numbers on the $y$-axis correspond to the number of perineuronal nets (PNN) counted in one hemisphere]. Notably, the extent of neuronal cell death of this particular subset of interneurones does not differ between terminally sick PV-/- and $\mathrm{PV}+/+$ mice. Open circles in the box-whisker plot represent outliers; ${ }^{* * *}$ indicates significance of differences at $P<0.001$. (B- D) Typical immunohistochemistry for $\mathrm{ILB}_{4}$ in the retrosplenial cortex of a scrapie infected PV-/- (C) as well as PV+/+ mouse (D) and in a mock inoculated $\mathrm{PV}+/+$ control mouse (B) demonstrating a substantial reduction of ILB 4 decorated PNN in the prion infected animals $(\mathbf{C}, \mathbf{D})$. Scale bar in (D) indicates $25 \mu \mathrm{m}$.

scrapie and mock inoculated $\mathrm{PV}+/+$ mice $(P=0.0003)$, as well as between scrapie and mock inoculated PV-/animals $(P=0.0006)$, but no significant difference between scrapie infected $\mathrm{PV}+/+$ and $\mathrm{PV}-/-$ animals $(P=0.63)$, or mock inoculated $P V+/+$ and $P V-/-$ mice $(P=0.13)$ respectively.

\section{No significant difference in Purkinje cell numbers between scrapie inoculated $\mathrm{CB}+/+$ and CB-/- mice}

In 20 microscopic high power fields (region A, defined in the Materials and methods section) and 12 high power fields (region B), we counted an average number of $5.0 \pm 2.2$ (region A) and $5.0 \pm 2.0$ (region B) Purkinje cells in CB-deficient mice (Figure 5A,C), and $4.7 \pm 2.1$ (region A) and $5.3 \pm 1.9$ (region B) Purkinje cells in controls (Figure 5A,B,D).

\section{Discussion}

The principal results of our study are: (i) deficiency for $\mathrm{CB}$ in scrapie inoculated mice results in a small, but highly significant reduction of survival time, but does not influence the general cerebral lesion profile, nor the extent of degeneration of cerebellar Purkinje cells, in the terminal stage of the disease; and (ii) deficiency for PV does not alter survival time, general cerebral lesion profile or degree of selective degeneration of cortical interneurones surrounded by PNN in mice experimentally challenged with scrapie prions.

Previous demonstrations of an early, selective and severe loss of inhibitory, GABAergic interneurones containing the calcium-buffer PV [5-7,17,18] raised speculations about a potential contribution of $\mathrm{PV}$ to the degeneration of this particular subset of interneurones by so far unknown mechanisms. The present study shows 

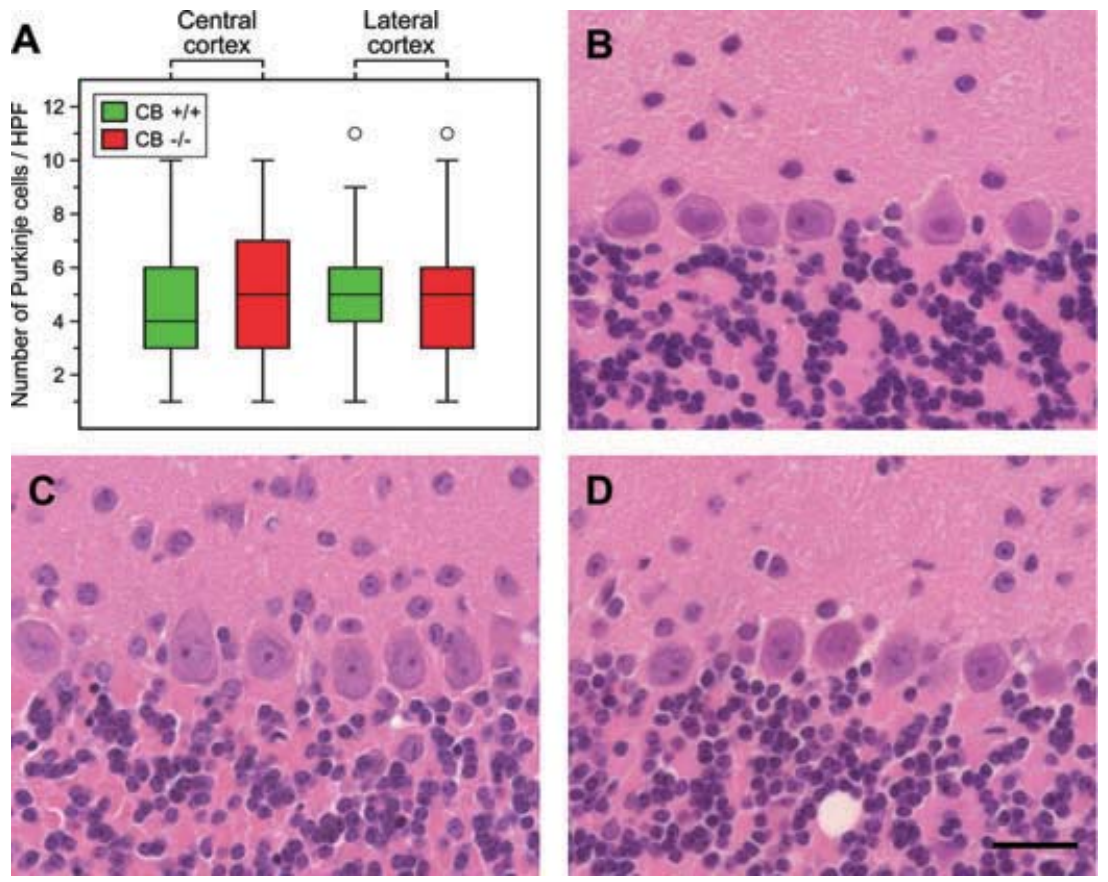

Figure 5. Survival of 'parvalbumin (PV) and calbindin D28k (CB)' coexpressing cerebellar Purkinje cells in CB-deficient mice and CB+/+ controls inoculated with 139A scrapie. (A) Comparable numbers of viable Purkinje cells are observed in the central (region A) and lateral (region $\mathrm{B}$ ) cerebellar cortex of scrapie inoculated $\mathrm{CB}-/-$ (red boxes) and $\mathrm{CB}+/+$ mice (green boxes). Notably, there is no selective degeneration of Purkinje cells in scrapie infected $\mathrm{CB}-/-$ mice as compared with prion inoculated $\mathrm{CB}+/+$ animals. Open circles in the box-whisker plot represent outliers. Details concerning analysed brain areas are stated in the Materials and methods section. (B-D) Representative photomicrographs (haematoxylin-eosin) of the cerebellar cortex illustrating a similar density of Purkinje cells in a scrapie infected CB-/(C) as well as $\mathrm{CB}+/+$ mouse (D), and in a mock inoculated $\mathrm{CB}+/+$ control animal (B). Scale bar in (D) corresponds to $25 \mu \mathrm{m}$.

that PV expression, at least at expression levels observed in wild-type mice, is not causally related to neurodegeneration in prion diseases. At the global level, PV-deficiency changes neither the survival time, nor the general lesion profile in scrapie inoculated animals (Figures 1A, 2A-C and 3A-F). And most importantly, the degeneration of vulnerable interneurones in murine scrapie is neither increased nor decreased in PV-deficient mice (Figure 4), implicating that $\mathrm{PV}$ deficiency does not protect from - and vice versa, PV expression does not contribute to - prioninduced neuronal cell death. This is also supported by the results obtained from prion inoculated $\mathrm{CB}-/-$ mice. In this particular mouse strain, cerebellar Purkinje cells contain high amounts of $\mathrm{PV}$ as the only, or at least major, remaining calcium binding protein known [10]; nevertheless, they are resistant to prion-related cell death to the same degree as in scrapie infected $\mathrm{CB}+/+$ controls (Figure 5) indicating again that $\mathrm{PV}$ expression per se does not promote prion-related neurodegeneration. It therefore seems that PV expression merely coexists with one or more factors responsible for selective neuronal vulnerability. It has been speculated that PNN, the specifically composed extracellular matrix around neocortical and hippocampal PV containing interneurones, might harbour such a factor contributing to the increased susceptibility, especially as Purkinje cells, which are not surrounded by PNN, are quite resistant against prion-related neurodegeneration [7]. In line with this idea, Belichenko et al. reported that the loss of PNN precedes the degeneration of PV-immunoreactive interneurones [17]; the authors concluded that PNN are destroyed due to an interaction between $\operatorname{PrP}^{\mathrm{C}}$ and/or $\mathrm{PrP}^{\mathrm{Sc}}$ and components of this extracellular matrix, and that they are finally replaced by $\mathrm{PrP}^{\mathrm{Sc}}$ deposits. This switch in cellular environment eventually initiates the death of PV-positive interneurones [17]. Another more recent report suggests that $\mathrm{PrP}^{\mathrm{sc}}$ does not interact directly with PNN, but withdraws a factor critical for prion conversion as well as for maintenance of this extracellular matrix, thereby destabilizing PNN. Subsequently, the nets degenerate, initiating again the loss of 
PV-containing interneurones [19]. Both hypotheses could explain the fact that the loss of PNN slightly precedes and exceeds the loss of PV-positive interneurones, but not the variability regarding the degree of survival or loss of nets and neurones: in experimental prion diseases, only about half of the PV-containing cortical interneurones surrounded by PNN degenerate, while the remaining survive until the animals reach the terminal disease stage. Thus, it may well be that PNN, like PV expression, only represent a surrogate marker for interneurones vulnerable to prion diseases, but that the death of these neurones is based on a still unknown pathomechanism.

Concerning the results obtained from CB knockout mice, the significantly shortened survival time of scrapie inoculated animals (Figure 1B) would be in accordance with a neuroprotective role of $\mathrm{CB}$, while unaltered cerebral lesion profiles (Figures 2D-F and 3G-M) and unchanged vulnerability of $\mathrm{CB}-/-$ Purkinje cells (Figure 5) in terminally sick mice do not support a neuroprotective function of this calcium-binding protein. On the other hand, the decreased survival time of $\mathrm{CB}-/-$ mice does not necessarily reflect a mere loss of neuroprotection. As was mentioned in the Introduction, CB knockout mice behave completely normally under physiological conditions in their home cage, whereas in specific locomotor tests, they show a significant impairment of Purkinje cell-dependent motor coordination resulting in an ataxic phenotype $[9,13]$. These observations have recently been confirmed in a Purkinje cell-specific CB null mutant mouse strain [20]. Impaired motor coordination is also a central part of the clinical picture observed in murine scrapie. Hence, the slightly shortened survival time of $\mathrm{CB}-/-$ mice might be explained by three scenarios: (i) a true, albeit small neuroprotective effect of $\mathrm{CB}$; as the expression pattern of $\mathrm{CB}$ is restricted to subpopulations of interneurones, this hypothetical loss of protection in $\mathrm{CB}-/-$ mice would have a limited impact only, explaining the fairly small difference in survival time between $\mathrm{CB}-/-$ and $\mathrm{CB}+/+$ animals; (ii) a mutual interaction between clinical scrapie and a 'stressinduced' $\mathrm{CB}-/-$ phenotype; in this case, synaptic damage and nerve cell dysfunction, both developing in the course of the prion disease, might be aggravated by motor coordination deficits due to $\mathrm{CB}$ deficiency; this could result in a more severe and more rapid disease phenotype, decreasing the time until the mice reach the terminal disease stage; and (iii) a combination of (i) and (ii). A cautious interpretation is also necessary regarding the unaltered number of surviving Purkinje cells in $\mathrm{CB}-/-$ mice. Several compensatory mechanisms induced in CB-/- Purkinje cells have been reported, which were discussed as homeostatic mechanisms of these neurones to cope with the absence of $\mathrm{CB}$ : (i) the length of spines was increased [21]; and (ii) the modelled $\mathrm{Ca}^{2+}$ extrusion rate in $\mathrm{CB}-/-$ dendritic spines had to be increased twofold in comparison with $\mathrm{CB}+/+$ spines in order to accurately fit the experimental data of spine $\mathrm{Ca}^{2+}$ transients, suggesting an up-regulation of proteins involved in $\mathrm{Ca}^{2+}$ extrusion [22]. Interestingly, no up-regulation of PV, the other prevalent $\mathrm{Ca}^{2+}$ buffer of Purkinje cells, was observed in cerebellar extracts of $\mathrm{CB}-/-$ mice [9]. Thus, the unchanged resistance of Purkinje cells of $\mathrm{CB}-/-$ mice against prion-induced cell death in this study does not allow the drawing of a general conclusion on CB's putative neuroprotective effect in prion diseases. However, in a recent study, a pronounced increase in corticosteroid levels was reported in the clinical stage of murine scrapie [23]. Elevated corticosteroid levels, in turn, are known to induce CB expression, at least in hippocampal neurones in rodents and primates [24-26] with a significant impact on selective neuronal survival in a rodent model of cerebral ischemia [27]. Of note, a majority of experiments (in vitro, but also in vivo) investigating the potential protective function of $\mathrm{CB}$ revealed a positive effect against excitotoxic stimuli (e.g. as discussed in [28-30]), while, on the other hand, studies using CB-deficient mice did not show any significant protection against neuronal injury [31-33].

Collectively, our current results studying scrapieinfected $\mathrm{CB}-/$ - mice do not permit the drawing of a final conclusion on a potential neuroprotective role of $\mathrm{CB}$ in prion diseases. The shortened survival time of scrapieinfected CB-/- mice is generally in accordance with a neuroprotective effect of $\mathrm{CB}$ but does not prove it due to the possible interference with a $\mathrm{CB}-/-$ phenotype; the unaltered lesion profiles in terminally sick $\mathrm{CB}-/-$ mice, on the other hand, do not corroborate a neuroprotective function of $\mathrm{CB}$, although the analysis of semiquantitative lesion profiles might be too insensitive to disclose minimal changes when used in a loss of function model restricted to a limited number of neurones only. Further experiments including the inoculation of mice ectopically (over)expressing $\mathrm{CB}$ in most neurones - on a constitutive or inducible basis - should allow to further dissect CB's possible protective influence on neuronal survival in prion diseases. 


\section{Acknowledgements}

The authors thank H. Flicker for excellent technical assistance. The project was supported by the Austrian Fund for Scientific Research (FWF, grant P14584-PSY to H.B), as well as the Deutsche Forschungsgemeinschaft (DFG, grant ME1121/3-3 to M.M), and is part of the framework of the European Network of Excellence 'NeuroPrion' (FOOD-CT2004-506579, Subproject: PRIOGEN).

\section{References}

1 Collinge J. Molecular neurology of prion disease. J Neurol Neurosurg Psychiatry 2005; 76: 906-19

2 Unterberger U, Voigtländer T, Budka H. Pathogenesis of prion diseases. Acta Neuropathol (Berl) 2005; 109: 3248

3 Weissmann C. The state of the prion. Nat Rev Microbiol 2004; 2: 861-71

4 Weissmann C. Birth of a prion: spontaneous generation revisited. Cell 2005; 122: 165-8

5 Guentchev M, Hainfellner JA, Trabattoni GR, Budka H. Distribution of parvalbumin-immunoreactive neurons in brain correlates with hippocampal and temporal cortical pathology in Creutzfeldt-Jakob disease. J Neuropathol Exp Neurol 1997; 56: 1119-24

6 Guentchev M, Groschup MH, Kordek R, Liberski PP, Budka H. Severe, early and selective loss of a subpopulation of GABAergic inhibitory neurons in experimental transmissible spongiform encephalopathies. Brain Pathol 1998; 8: 615-23

7 Guentchev M, Wanschitz J, Voigtländer T, Flicker H, Budka H. Selective neuronal vulnerability in human prion diseases. Fatal familial insomnia differs from other types of prion diseases. Am J Pathol 1999; 155: 1453-7

8 Schmidt H, Schwaller B, Eilers J. Calbindin D28k targets myo-inositol monophosphatase in spines and dendrites of cerebellar Purkinje neurons. Proc Natl Acad Sci USA 2005; 102: 5850-5

9 Airaksinen MS, Eilers J, Garaschuk O, Thoenen H, Konnerth A, Meyer M. Ataxia and altered dendritic calcium signaling in mice carrying a targeted null mutation of the calbindin D28k gene. Proc Natl Acad Sci USA 1997; 94: 1488-93

10 Schwaller B, Meyer M, Schiffmann S. 'New' functions for 'old' proteins: the role of the calcium-binding proteins calbindin D-28k, calretinin and parvalbumin, in cerebellar physiology. Studies with knockout mice. Cerebellum 2002; 1: 241-58

11 Schwaller B, Dick J, Dhoot G, Carroll S, Vrbova G, Nicotera P, Pette D, Wyss A, Bluethmann H, Hunziker W, Celio MR. Prolonged contraction-relaxation cycle of fasttwitch muscles in parvalbumin knockout mice. Am J Physiol 1999; 276: C395-403
12 Vreugdenhil M, Jefferys JG, Celio MR, Schwaller B. Parvalbumin-deficiency facilitates repetitive IPSCs and gamma oscillations in the hippocampus. J Neurophysiol 2003; 89: 1414-22

13 Farre-Castany MA, Schwaller B, Gregory P, Barski J, Mariethoz C, Eriksson JL, Tetko IV, Wolfer D, Celio MR, Schmutz I, Albrecht U, Villa AE. Differences in locomotor behavior revealed in mice deficient for the calciumbinding proteins parvalbumin, calbindin D-28k or both. Behav Brain Res 2007; 178: 250-61

14 Fraser H, Dickinson AG. The sequential development of the brain lesion of scrapie in three strains of mice. J Comp Pathol 1968; 78: 301-11

15 Bruce ME, McConnell I, Fraser H, Dickinson AG. The disease characteristics of different strains of scrapie in Sinc congenic mouse lines: implications for the nature of the agent and host control of pathogenesis. J Gen Virol 1991; 72: 595-603

16 Paxinos G, Franklin KBJ. The Mouse Brain in Stereotaxic Coordinates, 2nd edn. San Diego, CA: Academic Press 2001

17 Belichenko PV, Miklossy J, Belser B, Budka H, Celio MR. Early destruction of the extracellular matrix around parvalbumin-immunoreactive interneurons in Creutzfeldt-Jakob disease. Neurobiol Dis 1999; 6: 269-79

18 Ferrer I, Casas R, Rivera R. Parvalbuminimmunoreactive cortical neurons in Creutzfeldt-Jakob disease. Ann Neurol 1993; 34: 864-6

19 Moleres FJ, Velayos JL. Expression of $\operatorname{PrP}(C)$ in the rat brain and characterization of a subset of cortical neurons. Brain Res 2005; 1056: 10-21

20 Barski JJ, Hartmann J, Rose CR, Hoebeek F, Morl K, NollHussong M, De Zeeuw CI, Konnerth A, Meyer M. Calbindin in cerebellar Purkinje cells is a critical determinant of the precision of motor coordination. J Neurosci 2003; 23: 3469-77

21 Vecellio M, Schwaller B, Meyer M, Hunziker W, Celio MR. Alterations in Purkinje cell spines of calbindin D-28 k and parvalbumin knock-out mice. Eur J Neurosci 2000; 12: $945-54$

22 Schmidt H, Stiefel KM, Racay P, Schwaller B, Eilers J. Mutational analysis of dendritic $\mathrm{Ca} 2+$ kinetics in rodent Purkinje cells: role of parvalbumin and calbindin D28k. J Physiol 2003; 551: 13-32

23 Voigtländer T, Unterberger U, Touma C, Palme R, Polster B, Strohschneider M, Dorner S, Budka H. Prominent corticosteroid disturbance in experimental prion disease. Eur J Neurosci 2006; 23: 2723-30

24 Iacopino AM, Christakos S. Corticosterone regulates calbindin-D28k mRNA and protein levels in rat hippocampus. J Biol Chem 1990; 265: 10177-80

25 Krugers HJ, Medema RM, Postema F, Korf J. Regionspecific alterations of calbindin-D28k immunoreactivity in the rat hippocampus following adrenalectomy and corticosterone treatment. Brain Res 1995; 696: 89-96 
26 McMillan PJ, Wilkinson CW, Greenup L, Raskind MA, Peskind ER, Leverenz JB. Chronic cortisol exposure promotes the development of a GABAergic phenotype in the primate hippocampus. J Neurochem 2004; 91: 843-51

27 Rami A, Rabie A, Winckler J. Synergy between chronic corticosterone treatment and cerebral ischemia in producing damage in noncalbindinergic neurons. Exp Neurol 1998; 149: 439-46

28 Yenari MA, Minami M, Sun GH, Meier TJ, Kunis DM, McLaughlin JR, Ho DY, Sapolsky RM, Steinberg GK. Calbindin $\mathrm{d} 28 \mathrm{k}$ overexpression protects striatal neurons from transient focal cerebral ischemia. Stroke 2001; 32: 1028-35

29 D'Orlando C, Celio MR, Schwaller B. Calretinin and calbindin D-28k, but not parvalbumin protect against glutamate-induced delayed excitotoxicity in transfected N18-RE 105 neuroblastoma-retina hybrid cells. Brain Res 2002; 945: 181-90

30 Rintoul GL, Raymond LA, Baimbridge KG. Calcium buffering and protection from excitotoxic cell death by exogenous calbindin-D28k in HEK 293 cells. Cell Calcium 2001; 29: 277-87

31 Bouilleret V, Schwaller B, Schurmans S, Celio MR, Fritschy JM. Neurodegenerative and morphogenic changes in a mouse model of temporal lobe epilepsy do not depend on the expression of the calcium-binding proteins parvalbumin, calbindin, or calretinin. Neuroscience 2000; 97: 47-58

32 Airaksinen MS, Thoenen H, Meyer M. Vulnerability of midbrain dopaminergic neurons in calbindin-D28kdeficient mice: lack of evidence for a neuroprotective role of endogenous calbindin in MPTP-treated and weaver mice. Eur J Neurosci 1997; 9: 120-7

33 Klapstein GJ, Vietla S, Lieberman DN, Gray PA, Airaksinen MS, Thoenen H, Meyer M, Mody I. CalbindinD28k fails to protect hippocampal neurons against ischemia in spite of its cytoplasmic calcium buffering properties: evidence from calbindin-D28k knockout mice. Neuroscience 1998; 85: 361-73 